\title{
Método das Relações de Recorrência aplicado no problema do elétron em campo magnético constante e uniforme
}

\author{
Problem of a single electron in an external magnetic field solved via Method of Recurrence Relations \\ Luciana Caroline Saraiva Bispo ${ }^{1}$, Maria Eugênia Silva Nunes*1 \\ ${ }^{1}$ Universidade Federal de Ouro Preto, Departamento de Física, Ouro Preto, MG, Brasil
}

\begin{abstract}
Recebido em 16 de Novembro, 2017. Revisado em 21 de Dezembro, 2017. Aceito em 09 de Janeiro, 2018.
\end{abstract}
\begin{abstract}
Nesse trabalho utilizamos o Método das Relações de Recorrência (MRR) para estudar o comportamento de um elétron na presença de um campo magnético uniforme aplicado ao longo do eixo z de um sistema de referência estabelecido. Encontramos que as funções de autocorrelação temporais nas direções x e y, respectivamente $\mathrm{C}_{\mathrm{x}}(\mathrm{t})$ e $\mathrm{C}_{\mathrm{y}}(\mathrm{t})$, oscilam periodicamente no tempo, enquanto na direção $\mathrm{z}$, a função de autocorrelação temporal, $\mathrm{C}_{\mathrm{z}}(\mathrm{t})$, tem valor constante e igual a um, corroborando o resultado esperado de que o spin do elétron precessa em torno da direção de aplicação do campo magnético uniforme e independente do tempo.

Palavras-chave: Dinâmica de Spins, Método das Relações de Recorrência.
\end{abstract}

\begin{abstract}
We use the Method of Recurrence Relations (MRR) to study the behavior of a single electron in the presence of a uniform magnetic field along the z-direction. We find that the time-dependent autocorrelation functions in the $\mathrm{x}$ and y-directions, $\mathrm{C}_{\mathrm{x}}(\mathrm{t})$ and $\mathrm{C}_{\mathrm{y}}(\mathrm{t})$, respectively, oscillate periodically in time, while in the z-direction the temporal autocorrelation function $\mathrm{C}_{z}(\mathrm{t})$ has a constant value, corroborating the expected result that the spin precesses about the direction of the applied magnetic field.
\end{abstract}

Keywords: Spin Dynamics, Method of Recurrence Relations.

\section{Introdução}

Materiais magnéticos estão presentes no nosso dia a dia desde as aplicações mais simples como, por exemplo, os imãs de geladeira até as mais sofisticadas como armazenamento de informações em computadores. Atualmente, uma quantidade significativa de informações está gravada magneticamente em discos rígidos de computadores. A gravação das informações é feita utilizando-se o código binário (0 ou 1). Magneticamente, 0 poderia ser representado como spin orientado para baixo e 1 por spin orientado para cima em relação a algum sistema de referência. Hoje em dia, muitos pesquisadores trabalham na spintrônica (ou eletrônica de spins) [1] que visa controlar a corrente num dispositivo eletrônico, não somente pela carga do elétron, mas também pelo seu spin. Os objetivos principais são o de se aumentar a velocidade de processamento de informações e diminuir as perdas de energia nas transmissões de informação. Além disso, em Medicina, por exemplo, temos métodos de diagnóstico baseados em ressonância de spins, tanto eletrônicos (Espectroscopia de Ressonância Paramagnética Eletrônica) quanto nucleares (Ressonância Magnética Nuclear) [2,3]. Vê-se, por tudo isso, a importância do estudo de sistemas de spins.

*Endereço de correspondência: mariaeugenia@iceb.ufop.br
Existem muitas técnicas analíticas ou numéricas [4] para a obtenção de soluções exatas ou aproximadas para modelos de spins. Dentre essas técnicas temos o Método das Relações de Recorrência (MRR) [5,6,7], que é bastante útil na obtenção da dinâmica de sistemas de muitos corpos quânticos. Esse método permite obter informações apuradas para propriedades do sistema como, funções correlação, funções espectrais e correntes de spins, dentre outras. O MRR utiliza o processo Grarn-Schmidt de ortogonalização, o qual é bastante geral e permite a construção de um conjunto completo de vetores ortogonalizados do espaço de Hilbert. O método de relações de recorrência foi formulado por Howard Lee [8,9], como uma generalização do formalismo desenvolvido, dentre outros, por Mori [10,11].

\section{Spin do elétron e elétron em campo magnético constante e uniforme}

\subsection{Spin do elétron}

Entre 1921 e 1922, Otto Stern e Walther Gerlach realizaram um experimento que visava observar se havia quantização espacial do momento angular orbital dos elétrons em átomos [12]. O experimento basicamente consistia em fazer um feixe de átomos, inicialmente de prata, passar por um campo magnético não homogêneo ao longo de um eixo (escolhido como eixo z em relação ao referen- 
cial do laboratório) produzido por um imã e então medir a distribuição desses átomos em um anteparo na saída do imã. Eles observaram que, aproximadamente metade dos átomos colidia com uma extremidade da placa e a outra metade na posição simetricamente oposta, não registrando praticamente nenhum átomo em qualquer posição intermediaria. A física clássica não explicava tal resultado, uma vez que, submetido a um campo magnético, um momento magnético clássico exibe um espectro continuo de valores. Em 1927 Phipps e Taylor repetiram o experimento de Stern e Gerlach com átomos neutros e com momento angular orbital nulo. Era esperado que tais átomos passassem pela região de campo magnético não homogêneo sem sofrerem nenhuma deflexão. No entanto, eles observaram que o feixe também se dividia em dois ao passar pela região com campo magnético. Esses experimentos indicavam que os átomos possuíam uma espécie de momento magnético intrínseco (M) que não estava relacionado ao momento orbital dos elétrons. Portanto, deveria existir também um momento angular intrínseco, que foi chamado de spin e representado pelo vetor $\mathbf{S}$. A relação entre o momento magnético intrínseco e o momento angular intrínseco é dada por:

$$
\vec{M}=g \vec{S}
$$

A constante de proporcionalidade, g, é chamada razão giromagnética. O spin do elétron é um momento angular que existe independentemente do estado de movimento no qual a partícula se encontra. Cada partícula elementar tem um spin imutável associado a ela. Por exemplo, o spin do elétron é $1 / 2$, o spin dos fótons é 1 e o spin dos grávitons é 2 . Vale lembrar que partículas com spins semiinteiros são ditas férmions [12] e obedecem ao princípio de exclusão de Pauli. Já partículas com valores de spins inteiros são chamadas de bósons [12] e não precisam obedecer ao princípio de exclusão de Pauli.

Wolfgang Pauli postulou que um elétron podia ser modelado por um vetor bidimensional complexo e criou uma teoria de momento angular que atuasse neste espaço. $\mathrm{O}$ estado mais geral que pode ser associando a uma partícula de spin $1 / 2$, pode ser expresso como uma matriz coluna de dois elementos.

$$
\xi=\left(\begin{array}{c}
a \\
b
\end{array}\right)=\alpha \xi_{+}+\beta \xi_{-},
$$

em que o índice + representa uma matriz que descreve um spin apontando para cima ( $\uparrow)$ em relação a algum sistema de referência e o índice - representa o spin apontando para baixo $(\downarrow)$. Ou seja,

$$
\xi_{+}=\left(\begin{array}{l}
1 \\
0
\end{array}\right)=|\uparrow\rangle \quad \text { e } \quad \xi_{-}=\left(\begin{array}{l}
1 \\
0
\end{array}\right)=|\downarrow\rangle
$$

representam os estados acessíveis para o spin de uma partícula quântica. No que diz respeito ao estado de spin de uma partícula, são necessários dois números para caracterizar completamente o estado, são os números quânticos m (associado ao momento magnético) e o número quântico s (associado ao spin) que fornece a orientação espacial do momento magnético, ou seja, nos se informa se o spin aponta para cima ou para baixo em um dado sistema de referência. Para o elétron, $\mathrm{s}=1 / 2 \mathrm{e}$ $\mathrm{m}_{+}=+1 / 2,(\uparrow)$, enquanto $\mathrm{m}_{-}=-1 / 2,(\downarrow)$.

Na descrição de Pauli, as componentes do momento angular de spin são dadas por

$$
S_{x}=\frac{\hbar}{2} \sigma_{x}, \quad S_{y}=\frac{\hbar}{2} \sigma_{y} \quad \text { e } \quad S_{z}=\frac{\hbar}{2} \sigma_{z},
$$

onde $\hbar$ é a constante de Planck, h, dividida por $2 \pi$.

As matrizes $\sigma_{\mathrm{x}}, \sigma_{\mathrm{y}}$ e $\sigma_{\mathrm{z}}$ são ditas matrizes de Pauli com os seguintes valores:

$$
\begin{aligned}
\sigma_{x}=\left(\begin{array}{ll}
0 & 1 \\
1 & 0
\end{array}\right), \quad \sigma_{y}=\left(\begin{array}{cc}
0 & -i \\
i & 0
\end{array}\right) \\
\text { e } \quad \sigma_{z}=\left(\begin{array}{cc}
1 & 0 \\
0 & -1
\end{array}\right)
\end{aligned}
$$

Além disso, temos as seguintes relações de comutação para as componentes do momento angular:

$$
\begin{aligned}
& {\left[S_{x}, S_{y}\right]=i \hbar S_{z} \quad\left[S_{y}, S_{z}\right]=i \hbar S_{x} \quad\left[S_{z}, S_{x}\right]=i \hbar S_{y}} \\
& {\left[S_{y}, S_{x}\right]=-i \hbar S_{z} \quad\left[S_{z}, S_{y}\right]=-i \hbar S_{x} \quad\left[S_{x}, S_{z}\right]=-i \hbar S_{y}}
\end{aligned}
$$

\subsection{Eletron em campo magnético externo constante e uniforme}

Vimos anteriormente que os elétrons possuem momento magnético intrínseco $(\mathbf{M})$ relacionado ao seu momento angular intrínseco $(\mathbf{S})$.

$$
\vec{M}=g \vec{S}=g\left(S_{x} \hat{i}+S_{y} \hat{j}+S_{z} \hat{k}\right)
$$

Quando um dipolo magnético é posicionado em um campo magnético $\mathbf{B}$, o seu momento magnético tende a se alinhar com o campo e a menor energia de configuração do sistema ocorre quando o momento angular da partícula alinha-se com o campo externo.

No sistema estudado temos um elétron em repouso em um campo magnético uniforme que aponta para a direção z, ou seja,

$$
\vec{B}=B_{0} \hat{k}
$$

A energia associada à interação do elétron com o campo é, então, dada pelo hamiltoniano:

$$
H=-\vec{M} \cdot \vec{B}=-g\left(S_{x} \hat{i}+S_{y} \hat{j}+S_{z} \hat{k}\right) \cdot B_{0} \hat{k}=-g B_{0} S_{z}
$$

A forma matricial do hamiltoniano é obtida via matrizes de Pauli, 


$$
H=-g B_{0} S_{z}=-g B_{0} \frac{\hbar}{2} \sigma_{z}=-g B_{0} \frac{\hbar}{2}\left(\begin{array}{cc}
1 & 0 \\
0 & -1
\end{array}\right) .
$$

Sabemos que os autovetores (estados) e autovalores (no caso, energias) podem ser obtidos por

$$
H \xi_{+}=E_{+} \xi_{+} \quad \text { e } H \xi_{-}=E_{-} \xi_{-} .
$$

Os autovetores e autovalores são, então

$$
\begin{aligned}
& \xi_{+}=\left(\begin{array}{l}
1 \\
0
\end{array}\right) \quad \text { com } \quad E_{+}=-\left(\frac{g B_{0} \hbar}{2}\right), \\
& \xi_{-}=\left(\begin{array}{l}
0 \\
1
\end{array}\right) \quad \text { com } \quad E_{-}=+\left(\frac{g B_{0} \hbar}{2}\right) .
\end{aligned}
$$

A solução da equação de onda que descreve o sistema pode ser expressa em termos de estados estacionários:

$$
\begin{aligned}
& \xi(t)=\alpha \xi_{+} e^{-\frac{i E_{+} t}{\hbar}}+\beta \xi_{-} e^{-\frac{i E_{-} t}{\hbar}}=\alpha\left(\begin{array}{l}
1 \\
0
\end{array}\right) e^{\frac{i g B_{0} t}{2}}+ \\
& \beta\left(\begin{array}{l}
0 \\
1
\end{array}\right) e^{-\frac{i g B_{0} t}{2}}=\left(\begin{array}{c}
\alpha e^{\frac{i g B_{0} t}{2}} \\
\beta e^{-\frac{i g B_{0} t}{2}}
\end{array}\right) .
\end{aligned}
$$

Para calcular as constantes $\alpha$ e $\beta$ lembramos que a solução deve ser normalizada para qualquer tempo t e, em $\mathrm{t}=0$, temos

$$
\xi(0)=\left(\begin{array}{c}
\alpha \\
\beta
\end{array}\right)
$$

A condição de normalização nos diz que

$$
\langle\xi(0) \mid \xi(0)\rangle=1 \Rightarrow\left(\begin{array}{cc}
\alpha & \beta
\end{array}\right)\left(\begin{array}{l}
\alpha \\
\beta
\end{array}\right)=1 .
$$

Logo $|\alpha|^{2}+|\beta|^{2}=1 \mathrm{e}$

$$
\alpha=\cos \left(\frac{\theta}{2}\right) \quad \text { e } \beta=\operatorname{sen}\left(\frac{\theta}{2}\right),
$$

em que $\theta$ é um ângulo fixo.

Logo, temos que a solução da equação de onda dependente do tempo, para uma partícula de spin $1 / 2 \mathrm{em}$ um campo magnético uniforme:

$$
\xi(t)=\left(\begin{array}{c}
\cos \frac{\theta}{2} e^{i \frac{g B_{0} t}{2}} \\
\operatorname{sen} \frac{\theta}{2} e^{-i \frac{g B_{0} t}{2}}
\end{array}\right) .
$$

Calculando o valor esperado do vetor momento angular de spin $\mathbf{S}$, como uma função de tempo nós podemos obter informações úteis sobre a dinâmica desse sistema. $\mathrm{O}$ valor esperado de $<\mathbf{S}>$ é definido como

$$
\langle\vec{S}\rangle=\left\langle S_{x}\right\rangle \hat{i}+\left\langle S_{y}\right\rangle \hat{j}+\left\langle S_{z}\right\rangle \hat{k},
$$

com

$$
\begin{aligned}
& \left\langle S_{x}\right\rangle=\left\langle\xi(t)\left|S_{x}\right| \xi(t)\right\rangle,\left\langle S_{y}\right\rangle=\left\langle\xi(t)\left|S_{y}\right| \xi(t)\right\rangle \\
& \mathrm{e} \quad\left\langle S_{z}\right\rangle=\left\langle\xi(t)\left|S_{z}\right| \xi(t)\right\rangle
\end{aligned}
$$

Para a componente $\mathrm{x}$, teremos

$$
\begin{aligned}
& \left\langle S_{x}\right\rangle=\left(\cos \left(\frac{\theta}{2}\right) e^{-i \frac{g B_{0} t}{2}} \operatorname{sen}\left(\frac{\theta}{2}\right) e^{i \frac{g B_{0} t}{2}}\right) . \\
& {\left[\frac{\hbar}{2}\left(\begin{array}{ll}
0 & 1 \\
1 & 0
\end{array}\right)\left(\begin{array}{c}
\cos \left(\frac{\theta}{2}\right) e^{i \frac{g B_{0} t}{2}} \\
\text { en }\left(\frac{\theta}{2}\right) e^{-i \frac{g B_{0} t}{2}}
\end{array}\right)\right] .}
\end{aligned}
$$

O que leva a

$$
\left\langle S_{x}\right\rangle=\frac{\hbar}{2} \operatorname{sen}(\theta) \cos \left(g B_{0} t\right) .
$$

Analogamente,

$$
\left\langle S_{y}\right\rangle=-\frac{\hbar}{2} \operatorname{sen}(\theta) \operatorname{sen}\left(g B_{0} t\right) \text { e }\left\langle S_{z}\right\rangle=\frac{\hbar}{2} \cos (\theta) .
$$

Observamos então que as componentes x e y do momento angular de spin variam periodicamente no tempo, enquanto a componente $\mathrm{z}$ tem um valor constante. Representando o valor esperado de $<\mathbf{S}>$ em um sistema de coordenadas cartesiano, notamos que $<\mathbf{S}>$ está inclinado de um ângulo constante $\theta$ em relanão ao eixo z e precessiona em torno desse eixo com uma frequência $\omega=\mathrm{g} \mathrm{B}_{0}$. Essa frequência é a conhecida frequência de Larmor. Na Figura 1 apresentamos um diagrama do comportamento do vetor momento magnético de spin, $\mathbf{S}$, do elétron numa região do espaço com campo magnético uniforme e independente do tempo.

\section{Método das relações de recorrência}

Para estudarmos a dinâmica de um operador hermitiano (possui autovalores reais) em um determinado sistema físico, nós usaremos o Método das Relações de Recorrência (MRR). Esse método utiliza o processo GrarnSchmidt de ortogonalização para a construção de uma base vetorial no espaço de Hilbert d dimensional.

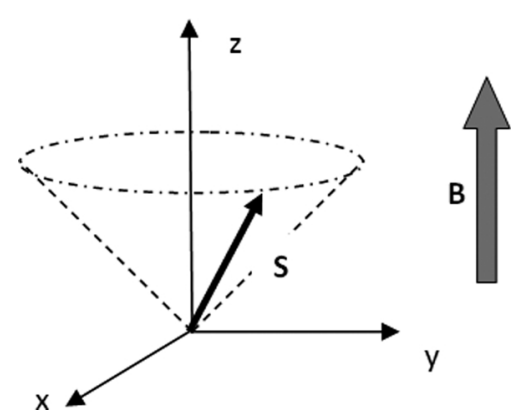

Figura 1: Precessão do momento angular total de spin $\mathbf{S}$ em torno da direção de aplicação do campo magnético externo uniforme. $\mathrm{O}$ ângulo formado entre o vetor $\mathbf{S}$ e o eixo $z$ é igual a $\theta$. 
Neste trabalho estamos interessados no cálculo das funções de autocorrelação temporais das variáveis dinâmicas $\sigma_{\mathrm{x}}, \sigma_{\mathrm{y}}$ e $\sigma_{\mathrm{z}}$, ou seja, queremos saber como as funções de autocorrelação das matrizes de Pauli comportam-se quando a partícula é submetida a um campo externo. Para obtermos essas informações utilizaremos o MRR, o qual é descrito abaixo.

Seja A um operador correspondente a um observável e $\mathrm{H}$ o hamiltoniano que descreve o sistema. Na representação de Heisenberg, a dependência temporal está no operador e, não na função de onda. Portanto, o operador A na descrição de Heisenberg toma a seguinte forma

$$
A(t)=e^{i H t} A(0) e^{-i H t},
$$

na qual se fez $\hbar=1$ por questões de simplicidade.

A evolução temporal de A é governada pela equação de Liouville

$$
\frac{d}{d t} A(t)=i L A(t)
$$

Na Eq. 25, L é o operador de Liouvile, que é definido como

$$
L A(t)=[H, A(t)]=H A(t)-A(t) H .
$$

No método das relações de recorrência, A(t) é considerado um vetor definido em um espaço de Hilbert, S, de dimensão d. A dimensionalidade do sistema dependerá do sistema tratado, bem como da variável dinâmica que se pretende estudar. O produto escalar (ou produto interno) em $\mathrm{S}$ é definido por meio do produto de Kubo,

$$
(X, Y)=\frac{1}{\beta} \int_{0}^{\beta} d \lambda\left\langle X(\lambda) Y^{\dagger}\right\rangle-\langle X\rangle\left\langle Y^{\dagger}\right\rangle,
$$

onde X e Y são vetores, definidos no espaço de Hilbert e os símbolos $<>$ representam médias térmicas no ensemble canônico. Além disso, $\beta=1 /\left(\mathrm{k}_{\mathrm{B}} \mathrm{T}\right)$, em que $\mathrm{T}$ é a temperatura e $\mathrm{k}_{\mathrm{B}}$ é a constante de Boltzmann. As médias térmicas no ensemble canônico são tomadas da seguinte maneira:

$$
\langle X\rangle=\frac{\operatorname{Tr} e^{-\beta H} X}{\operatorname{Tr} e^{-\beta H}} .
$$

Na representação de Heisenberg,

$$
X(\lambda)=e^{\lambda H} X e^{-\lambda H} .
$$

No limite de altas temperaturas $(\mathrm{T} \rightarrow \infty)$, o produto escalar $(\mathrm{X}, \mathrm{Y})$ reduz-se a

$$
(X, Y)=\frac{\operatorname{Tr} X Y^{\dagger}}{\operatorname{Tr} 1}
$$

em que Tr1 é o número de estados do sistema.

A quantidade de interesse $\mathrm{A}(\mathrm{t})$ é dada como uma expansão em termos de vetores de uma base ortogonalizada, não normalizada, $\left\{\mathrm{f}_{\nu}\right\}$ no espaço de Hilbert $\mathrm{S}$,

$$
A(t)=\sum_{\nu=0}^{d-1} a_{\nu}(t) f_{\nu}
$$

Sabemos que (Eq. 25),

$$
\left.\frac{d}{d t} A(t)\right|_{t=0}=\left.i L A(t)\right|_{t=0}=i L A(0) .
$$

Para verificar a relação de recorrência que gera a base de vetores $\mathrm{f}_{\nu}$, começamos definindo o primeiro vetor de base $(\nu=0)$ como

$$
\left.\left|f_{1}\right\rangle=i L\left|f_{0}\right\rangle=\| \frac{d}{d t} f_{0}(t)\right\rangle\left.\right|_{t=0} .
$$

Os vetores devem ser ortogonais, então os produtos escalares entre vetores diferentes deverão ser nulos. Portanto, $<\mathrm{f}_{0}\left|\mathrm{f}_{2}>=<\mathrm{f}_{1}\right| \mathrm{f}_{2}>=0$. O segundo vetor pode ser escrito como:

$$
\left|f_{2}\right\rangle=i L\left|f_{1}\right\rangle+|x\rangle .
$$

Aplicando as condições de ortogonalidade teremos,

$$
\left\langle f_{0} \mid f_{2}\right\rangle=\left\langle f_{0}|i L| f_{1}\right\rangle+\left\langle f_{0} \mid x\right\rangle=0 \text {. }
$$

Como

$$
\left\langle f_{0}\right| i L=\left\langle f_{1}\right|,
$$

obtemos,

$$
-\left\langle f_{1} \mid f_{1}\right\rangle+\left\langle f_{0} \mid x\right\rangle=0
$$

De maneira análoga,

$$
\left\langle f_{1} \mid f_{2}\right\rangle=\left\langle f_{1}|i L| f_{1}\right\rangle+\left\langle f_{1} \mid x\right\rangle=0 \Longrightarrow\left\langle f_{1} \mid x\right\rangle=0 .
$$

Vamos supor que

$$
x=c f_{0} .
$$

Levando a Eq. (39) na Eq.(37), vemos que,

$$
-\left\langle f_{1} \mid f_{1}\right\rangle+\left\langle f_{0} \mid c f_{0}\right\rangle=0
$$

e ainda,

$$
c=\frac{\left\langle f_{1} \mid f_{1}\right\rangle}{\left\langle f_{0} \mid f_{0}\right\rangle} \equiv \Delta_{1} .
$$

Portanto o segundo vetor da base (Eq. 34) fica dado por

$$
\left|f_{2}\right\rangle=i L\left|f_{1}\right\rangle+\Delta_{1}\left|f_{0}\right\rangle \text {. }
$$

Os outros vetores da base são construídos maneira análoga, observando-se a ortogonalidade entre os mesmos. Os vetores $\mathrm{f}_{\nu}$ podem ser construídos sistematicamente e satisfazem a seguinte relação de recorrência, dita Relação de Recorrência I (RRI), 


$$
f_{\nu+1}=i L f_{\nu}+\Delta_{\nu} f_{\nu-1} \quad 0 \leq \nu \leq d-1,
$$

em que

$$
\Delta_{\nu}=\frac{\left(f_{\nu}, f_{\nu}\right)}{\left(f_{\nu-1}, f_{\nu-1}\right)}
$$

com as seguintes definições,

$$
f_{-1} \equiv 0 \quad \text { e } \quad \Delta_{0} \equiv 1 .
$$

Podemos fazer uma escolha arbitrária para o primeiro vetor de base $\mathrm{f}_{0}$. Em geral, escolhe-se $\mathrm{f}_{0}=\mathrm{A}(0)$ e assim as condições iniciais impõem que $\mathrm{a}_{0}(0)=1$ e $\mathrm{a}_{\nu}(0)=0$ para valores de $\nu$ maiores ou iguais a 1 .

Isso pode ser verificado facilmente, pois

$$
A(t)=a_{0}(t) f_{0}+a_{1}(t) f_{1}+\ldots+a_{d-1}(t) f_{d-1} .
$$

E quando $\mathrm{t}=0$, temos,

$$
A(0)=a_{0}(0) f_{0}+a_{1}(0) f_{1}+\ldots+a_{d-1}(0) f_{d-1}=f_{0} .
$$

A função $a_{0}(t)$ representa a função de relaxação da teoria de resposta linear. No limite de altas temperaturas, $\mathrm{a}_{0}(\mathrm{t})$ é simplesmente a função de autocorrelação dependente do tempo $\mathrm{a}_{0}(\mathrm{t})=\mathrm{C}(\mathrm{t})$. A função de autocorrelação temporal para o operador A(t) nos informa sobre a correlação do operador consigo mesmo em relação ao tempo $\mathrm{t}=0$ :

$$
C(t)=\left\langle\frac{A(t) A(0)}{A(0) A(0)}\right\rangle=\frac{(A(t), A(0))}{(A(0), A(0))} .
$$

Expandindo A(t) na base de vetores apropriada, vemos que

$$
\begin{aligned}
& C(t)=\frac{(A(t), A(0))}{(A(0), A(0))} \\
& =\quad \frac{\left(a_{0}(t) f_{0}+a_{1}(t) f_{1}+\ldots, f_{0}\right)}{\left(f_{0}, f_{0}\right)}=a_{0}(t)
\end{aligned}
$$

Como a expansão de $\mathrm{A}(\mathrm{t})$ em termos dos vetores de base $\mathrm{f}_{\nu}$ satisfaz a equação de Liouville, o uso da relação de recorrência dá origem a uma segunda relação de recorrência para os $\mathrm{a}_{\nu}(\mathrm{t})$, dita Relação de Recorrência II (RRII):

$$
\Delta_{\nu+1} a_{\nu+1}(t)=-\frac{d}{d t} a_{\nu}(t)+a_{\nu-1}(t) \quad 0 \leq \nu \leq d-1
$$

em que $a_{-1}(t) \equiv 0$.

Note que os $\Delta_{\nu}$ são os únicos elementos que entram na construção dos $a_{\nu}(t)$, os quais determinam completamente a evolução temporal do operador.
Uma consequência de RRII é que a função de autocorrelação temporal $\mathrm{a}_{\mathrm{o}}(\mathrm{t})$ tem derivada nula na origem, isto é,

$$
\left.\frac{d}{d t} a_{0}(t)\right|_{t=0}=0
$$

o que impede, por exemplo, decaimento exponencial simples como solução exata para $\mathrm{a}_{\mathrm{o}}(\mathrm{t})$.

Da relação de recorrência RRII, vemos que

$$
\nu=0 \quad \Delta_{1} a_{1}=-\frac{d}{d t} a_{0}(t) .
$$

Vamos aplicar agora a transformada de Laplace à Eq. 52

$$
\int_{0}^{\infty} e^{-z t} \Delta_{1} a_{1}(t) d t=-\int_{0}^{\infty} e^{-z t} \frac{d a_{0}(t)}{d t} d t
$$

Integrando por partes o lado direito da equação, obtemos

$$
\Delta_{1} a_{1}(z)=1-z a_{0}(z)
$$

Repetindo o processo, ou seja, aplicando a transformada de Laplace aos termos com $\nu=1,2,3$, obtemos

$$
\Delta_{\nu+1} a_{\nu+1}(z)=a_{\nu-1}(z)-z a_{\nu}(z) \quad \nu \geq 1
$$

Partindo das relações de recorrência RRI e RRII, podemos escrever $\mathrm{a}_{0}(\mathrm{z})$ na forma de uma fração continuada:

$$
a_{0}(z)=\frac{1}{z+\frac{\Delta_{1}}{z+\frac{\Delta_{2}}{z+\ldots}}} .
$$

A função de autocorrelação temporal, $\mathrm{a}_{0}(\mathrm{t})$, é determinada aplicando-se a transformada de Laplace inversa em $\mathrm{a}_{0}(\mathrm{z})$.

$$
a_{0}(t)=L^{-1}\left(a_{0}(z)\right)
$$

Quando utilizamos o método das relações de recorrência, calculamos primeiro os vetores da base e, a seguir, os deltas. Vimos acima que os deltas determinam $\mathrm{a}_{0}(\mathrm{z}) \mathrm{e}$, por conseguinte, a função de autocorrelação temporal.

\section{Aplicação do Método das Relações de Recorrência no problema do elétron em um campo magnético externo uniforme}

Pretendemos obter o comportamento dinâmico das componentes do momento angular do spin de um elétron imerso em um campo magnético uniforme que aponta na direção z de um sistema de coordenadas. 


\subsection{Função de autocorrelação temporal na direção y}

A princípio obteremos os vetores da base e os deltas para posteriormente calcularmos as funções de autocorrelação temporais. Para o cálculo da função de autocorrelação temporal na direção y, escolhemos

$$
f_{0}=\sigma_{y} .
$$

A relação de recorrência RRI nos diz como proceder para encontrar o segundo vetor da base $f_{1}$ :

$$
f_{1}=i L f_{0}+\Delta_{0} f_{-1}=i L f_{0}=i\left[H, f_{0}\right]=i\left[H, \sigma_{y}\right] \text {, }
$$

já que $\mathrm{f}_{-1}=0$ e $\Delta_{0}=0$.

Como vimos na seção 2, o hamiltoniano para este problema é dado por:

$$
H=-\frac{g B_{0}}{2} \sigma_{z}
$$

Na qual se fez $\hbar=1$ por uma questão de coerência com o MRR.

Logo,

$$
\begin{array}{r}
f_{1}=i\left[-\frac{g B_{0}}{2} \sigma_{z}, \sigma_{y}\right]=-i \frac{g B_{0}}{2}\left[\sigma_{z}, \sigma_{y}\right]= \\
-i \frac{g B_{0}}{2}\left(-2 i \sigma_{x}\right)=-g B_{0} \sigma_{x} .
\end{array}
$$

Para o cálculo de $\Delta_{1}$, utilizamos

$$
\Delta_{1}=\frac{\left(f_{1}, f_{1}\right)}{\left(f_{0}, f_{0}\right)}=\frac{\left(-g B_{0} \sigma_{x},-g B_{0} \sigma_{x}\right)}{\left(\sigma_{y}, \sigma_{y}\right)}=\left(g B_{0}\right)^{2} .
$$

Para calcular o próximo vetor da base, devemos fazer $\nu=1$ na RRI:

$$
\begin{gathered}
f_{2}=i L f_{1}+\Delta_{1} f_{0}=i\left[-\frac{g B_{0}}{2} \sigma_{z},-g B_{0} \sigma_{y}\right]+ \\
\left(g B_{0}\right)^{2} \sigma_{y}=-\left(g B_{0}\right)^{2} \sigma_{y}+\left(g B_{0}\right)^{2} \sigma_{y}=0 .
\end{gathered}
$$

Como consequência,

$$
\Delta_{2}=\frac{\left(f_{2} f_{2}\right)}{\left(f_{1}, f_{1}\right)}=0 .
$$

Pode-se mostrar utilizando-se RRI que $\mathrm{f}_{3}=\mathrm{f}_{4}=\ldots=$ $\mathrm{f}_{\mathrm{d}-1}=0$. Além disso, $\Delta_{3}=\Delta_{4=\cdots=} \Delta_{\mathrm{d}-1}=0$.

Logo podemos calcular o valor de $\mathrm{a}_{0}(\mathrm{z})$,

$$
a_{0}(z)=\frac{1}{z+\frac{\Delta_{1}}{z+0}}=\frac{1}{z+\frac{\left(g B_{0}\right)^{2}}{z}}=\frac{z}{z^{2}+\left(g B_{0}\right)^{2}} .
$$

A função de autocorrelação temporal é então obtida tomando-se a transforma de Laplace inversa de $\mathrm{a}_{0}(\mathrm{z})$,

$$
a_{0}(t)=L^{-1} a_{0}(z)=\cos \left(\frac{g B_{0}}{2} t\right)=C_{y}(t) .
$$

Portanto, $\mathrm{a}_{0}(\mathrm{t})$ pode assumir valores no intervalo de -1 a 1 na direção do eixo y.

\subsection{Função de autocorrelação temporal na direção $x$}

O problema na direção x é simétrico ao problema em y. Para se fazer os cálculos basta seguir a mesma sequência apresentada na seção 4.1. Pode-se mostrar que, para $\mathrm{f}_{0}=\sigma_{\mathrm{x}}$

$$
a_{0}(t)=L^{-1} a_{0}(z)=\cos \left(\frac{g B_{0}}{2} t\right)=C_{x}(t) .
$$

\subsection{Função de autocorrelação temporal na direção $\mathrm{z}$}

Para direção z, escolhemos o primeiro vetor da base como

$$
f_{0}=\sigma_{z},
$$

o que leva a

$f_{1}=i\left[-\frac{g B_{0}}{2} \sigma_{z}, \sigma_{z}\right]=-i \frac{g B_{0}}{2}\left[\sigma_{z}, \sigma_{z}\right]=-i \frac{g B_{0}}{2}(0)=0$

e $\Delta_{1}=0$. Além disso, $\mathrm{f}_{2}=\mathrm{f}_{3}=\ldots=\mathrm{f}_{\mathrm{d}-1}=0$ e $\Delta_{2}=$ $\Delta_{3=\cdots=} \Delta_{\mathrm{d}-1}=0$.

Portanto,

$$
a_{0}(z)=\frac{1}{z}
$$

com tranfomada de Laplace inversa,

$$
a_{0}(t)=L^{-1}\left(\frac{1}{z}\right)=1=C_{z}(t),
$$

ou seja, a função de autocorrelação temporal na direção de aplicação do campo apresenta valor constante. O momento angular de spin $\mathrm{S}_{\mathrm{z}}$ está sempre positivamente correlacionado consigo mesmo, para todo tempo $t$.

\section{Considerações finais}

Com a aplicação do método de relação de recorrência (MRR), conseguimos obter o comportamento dinâmico em três dimensões do spin do elétron quando submetido ao campo magnético uniforme. Verificamos que na direção do campo (eixo z) a componente do spin, $\mathrm{S}_{\mathrm{z}}$, possui uma função de autocorrelação temporal com valor constante e igual a 1 , ou seja, $\mathrm{C}_{\mathrm{z}}(\mathrm{t})=1$. Porém, nos eixos $\mathrm{x}$ e $\mathrm{y}$, verificou-se que essas funções oscilam periodicamente no tempo e podem assumir valores que vão desde -1 a 1. Este resultado era esperado, pois como vimos no cálculo 
dos valores esperados dos spins, $S_{x}$ e $S_{y}$ são funções periódicas do tempo, enquanto $S_{z}$ apresenta um valor constante, ou seja, a componente $\mathrm{z}$ do spin está sempre positiva e integralmente correlacionada consigo mesma.

\section{Referências}

[1] C. Chappert, A. Fert, and F.N. Van Dau, Nature Materials 6, 813 (2007).

[2] A.P. Guimarães, Revista Brasileira de Ensino de Física 22, 360 (2000).

[3] C.M.T. Dieguez, L.V. Montanheiro, L.B. Cleto, L.B.M.J.C. Bonfim and C.A. Dartora, Revista Brasileira de Ensino de Física 40, e1310 (2018).

[4] J.B. Parkinson and D.J.J. Farnell, An Intoduction to Quantum Spin Systems. Lec. Notes Phys. 816 (Springer, Berlin, 2010).

[5] G. Muller, and V.S. Viswanath, The Recursion Method: Application to Many-Body Dynamics (Springer-Verlag, Berlin, 1994).

[6] J. Florencio and M.H. Lee, Physical Review B 35, 1835 (1987).

[7] M.E.S. Nunes, J.A. Plascak and J. Florencio, Physica A 332, 1 (2004).

[8] M.H. Lee, Phys. Rev. Lett. 49, 1072 (1982).

[9] M.H. Lee, Phys. Rev. B 26, 2547 (1982).

[10] H. Mori, Progress of Theoretical Physics 34, 399 (1965).

[11] H. Mori, Progress of Theoretical Physics 33, 423 (1965).

[12] D.J. Griffiths, Mecânica Quântica (Pearson Prentice Hall, São Paulo, 2011). 